\title{
Sidelobe-Free Beam-Steering using Optical Phased Arrays for Neural Probes
}

\author{
Fu-Der Chen ${ }^{1,2^{*}}$, Youngho Jung ${ }^{1}$, Tianyuan Xue ${ }^{2}$, Jason C. C. Mak ${ }^{2}$, Xianshu Luo ${ }^{3}$, Patrick Guo-Qiang Lo ${ }^{3}$, \\ Michael L. Roukes ${ }^{4}$, Joyce K. S. Poon ${ }^{1,2}$, and Wesley D. Sacher ${ }^{* * *}$ \\ ${ }^{I}$ Max Planck Institute of Microstructure Physics, Weinberg 2, 06120, Halle, Germany \\ ${ }^{2}$ Department of Electrical and Computer Engineering, University of Toronto, 10 King's College Rd., Toronto, Ontario M5S 3G4, Canada \\ ${ }^{3}$ Advanced Micro Foundry Pte Ltd, 11 Science Park Road, Singapore Science Park II, 117685, Singapore \\ ${ }^{4}$ Division of Physics, Mathematics, and Astronomy, California Institute of Technology, Pasadena, California 91125, USA \\ *fdchen@mpi-halle.mpg.de, **wesley.sacher@mpi-halle.mpg.de
}

\begin{abstract}
We demonstrate implantable neural probes with integrated silicon nitride optical phased arrays (OPAs) fabricated on $200 \mathrm{~mm}$ wafers for wavelengths near $480 \mathrm{~nm}$. A free-propagation slab enables the emission of a single steerable beam from an OPA. (C) 2021 The Author(s)
\end{abstract}

\section{Introduction}

Technologies for delivering patterned dynamic illumination at depth within the brain are critical to fully realizing the potential of optogenetics for studying the functionality of neural circuits. Optical stimulation (via optogenetic actuators) and activity readout of neurons (via genetically encoded calcium and voltage indicators) are common and widely deployed techniques for brain activity mapping [1,2]. Conventionally, light delivery into the brain relies on benchtop microscopes [2], miniaturized microscopes with GRIN lenses [3], and fiber optics [4]; with limitations due to the size of the optics and/or reconfigurability of the illumination. Recent demonstrations have shown that implantable silicon $(\mathrm{Si})$ probes with integrated photonic waveguides and small form factors can deliver patterned illumination at depth in brain tissue [5,6]. These neural probes use discrete, addressable, grating couplers that emit microscopic collimated beams. To enable steering of the grating-emitted beams, we have demonstrated a neural probe with multiple addressable optical phased arrays [7], where the emission beam angle is modulated by the input wavelength [7]. The work in [7] was limited by the presence of multiple beams (grating orders) emitted by each OPA, a common characteristic of conventional OPAs designed to mitigate inter-waveguide crosstalk by using an array pitch larger than half the wavelength $[8,9]$. The multiple beams limit the ability to independently address small volumes of brain tissue. Here, we report implantable neural probes with a novel OPA design that emits a single steerable beam over most of the steering range.

\section{OPA Design and Fabrication}

The OPA design and operation are shown in Fig. 1(a). The OPAs consist of $200 \mathrm{~nm}$ thick silicon nitride (SiN) waveguides. The design targets blue wavelengths from 460 - $492 \mathrm{~nm}$ for compatibility with the commonly used actuator Channelrhodopsin-2 and calcium indicator GCaMP6. As in [7], beam-steering is accomplished by wavelength tuning of the input light. Light in the OPA input waveguide is split into an array of 16 single-mode delay line waveguides (differential path length of $5 \mu \mathrm{m}$ ) via a star coupler. The 16 waveguides are subsequently brought to a 400 $\mathrm{nm}$ pitch, forming an end-fire phased array that emits an in-plane steerable beam into the free-propagation region

a

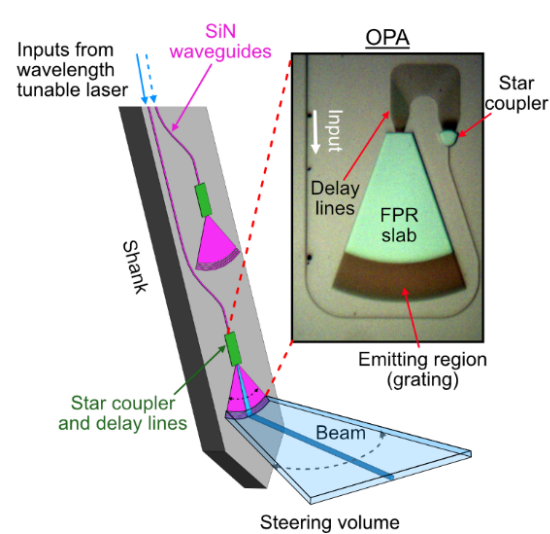

b

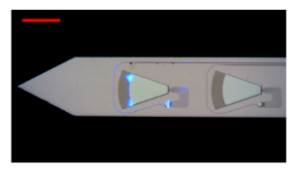

$\lambda=468 \mathrm{~nm}$

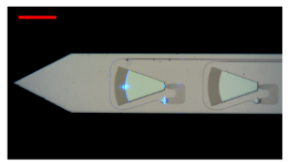

$\lambda=480 \mathrm{~nm}$

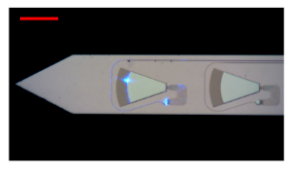

$\lambda=472 \mathrm{~nm}$

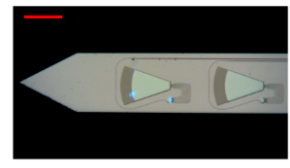

$\lambda=488 \mathrm{~nm}$
$\mathrm{C}$

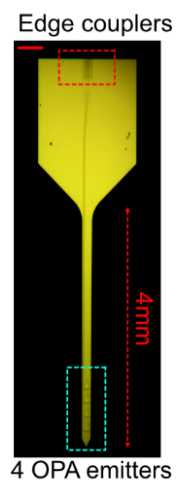

Fig. 1. OPA design and neural probes. (a) Illustration of the OPA design and operation. A steered beam in the free-propagation region (FPR) slab is emitted by the grating. Inset: annotated optical micrograph of one of the OPAs. (b) Optical micrographs of an OPA integrated onto a neural probe at various input wavelengths $(\lambda)$ showing beam steering in the FPR slab. (c) Optical micrograph of the OPA neural probe. The scale bars are (b) $100 \mu \mathrm{m}$ and (c) $400 \mu \mathrm{m}$. 


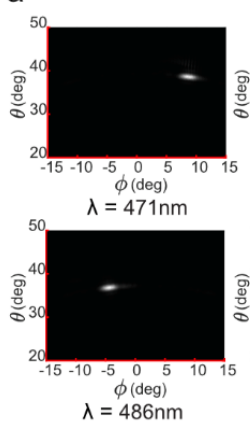

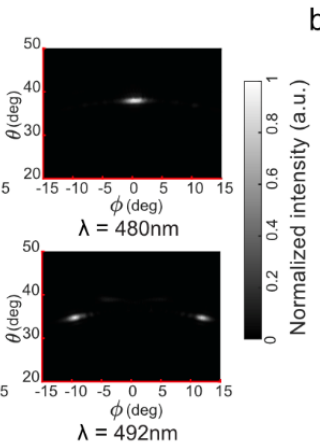

b

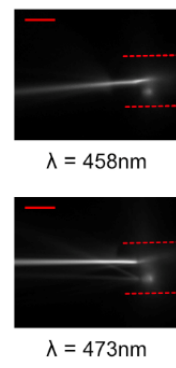

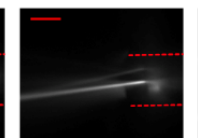

$\lambda=462 \mathrm{~nm}$

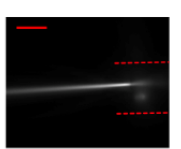

$\lambda=478 \mathrm{~nm}$

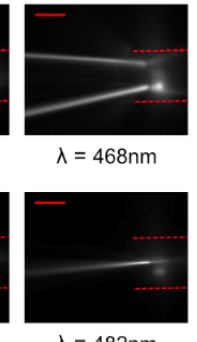

$\lambda=482 \mathrm{~nm}$ $c$

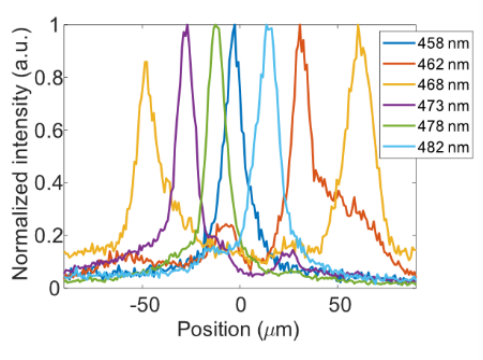

Fig. 2. Characterization of OPAs. (a) Measured far-field emission profile of the OPA on a test chip at various wavelengths $(\lambda)$. $\varphi$ is the lateral steering angle in Fig. 1(a) and $\theta$ is the coupling angle of the grating. $\varphi=0^{\circ}, \theta=0^{\circ}$ corresponds to the normal of the shank plane. (b) Measured top-down intensity beam profiles of OPA on a probe in fluorescein, and (c) vertical line-cuts of the fluorescein beam profile images in (b) at a horizontal propagation distance of $100 \mu \mathrm{m}$ from the OPA at multiple wavelengths. The scale bar in (b) is $100 \mu \mathrm{m}$ and the red dashed lines correspond to edges of the shank. All beam profiles have been normalized to the maximum intensity in each image.

(FPR) slab waveguide, Fig. 1(b). The steerable beam is radiated out of the plane by a curved grating at the end of the FPR slab (period $=480 \mathrm{~nm}$, duty cycle $=50 \%$ ). Steering of the emitted beam is a result of: 1 ) lateral translation of the beam along the grating, and 2) the dependence of the output coupling angle on the incident angle of the slab beam on the curved grating. The end-fire phased array at the input to the FPR slab enables low inter-waveguide crosstalk despite a small waveguide pitch. Additional grating orders of the end-fire phased array propagate at sufficiently large angles to intersect the slab sidewalls such that only a single grating order reaches the grating to be emitted.

Neural probes integrating 4 OPAs onto a single shank, Fig. 1(c), were fabricated at Advanced Micro Foundry on $200 \mathrm{~mm}$ Si wafers. The fabrication follows the method in [6] using plasma enhanced chemical vapor deposition (PECVD) for the SiN waveguide layer, deep ultraviolet lithography for patterning, and back-grinding for thinning the wafers to the probe thickness of $\approx 100 \mu \mathrm{m}$. Each OPA is connected to an edge coupler for fiber coupling, which enables spatial addressing of the different OPAs using a fiber image bundle [6]. The shank length is $4 \mathrm{~mm}$. The measured free spectral range (FSR) of the OPAs was about $23 \mathrm{~nm}$ near $\lambda=470 \mathrm{~nm}$. The pitch between the OPAs is $240 \mu \mathrm{m}$.

\section{Characterization}

The emission profiles of the OPAs were characterized in air and in a fluorescein solution. Wavelength tunable light from a supercontinuum laser coupled to a tunable bandpass filter was edge-coupled to the OPAs via a single-mode fiber. The far-field emission profiles of an OPA on a test chip, not a neural probe, were characterized using a Fourier imaging system angled at $\theta=33^{\circ}$ with a $\pm 24.8^{\circ}$ measurement range [9], Fig. 2(a). The test chip OPA was nominally identical to the neural probe OPA and had a waveguide array pitch at the FPR slab input of $360 \mathrm{~nm}$ and a grating period of $520 \mathrm{~nm}$. Continuous beam steering was observed over an angular range of $23.7^{\circ}$, and a single lobe was observed over a steering range of about $16^{\circ}$. At the far ends of the steering range, 2 lobes were present. Far-field measurements quantify the steering of the emitted beam angle but do not measure the lateral translation of the beam along the grating in the FPR slab. To quantify the full steering range of the beam, neural probes were immersed in fluorescein and top-down fluorescence images of the beam profiles were taken [6]; the probes were angled such that the beams were parallel to the fluid surface. The combination of lateral translation of the beam and changes in the emission angle are evident in the fluorescein beam profiles in Fig. 2(b). Fig. 2(c) shows a single lobe is steered over a lateral distance of $57 \mu \mathrm{m}$ at a distance of $100 \mu \mathrm{m}$ from the OPA. In contrast to our previous OPA neural probe design [7], a single beam is emitted over about $68 \%$ of the steering range. The steering range may be increased by optimization of the grating curvature, FPR slab, and array pitch at the FPR input.

\section{Conclusion}

In conclusion, we have presented implantable neural probes with multiple addressable OPAs, each capable of emitting a single steerable beam. The novel OPA design here provides new possibilities for increasing the complexity and utility of patterned illumination from neural probes for optogenetics and functional imaging [10].

Acknowledgments: The authors thank Dr. A. Stalmashonak for experimental assistance.

\section{References}

[1] K. Deisseroth, Nat. Neurosci. 18, 1213-1225 (2015).

[2] A. M. Packer et al., Nat. Methods 12, 140-146 (2015)

[3] K. K. Ghosh et al., Nat. Methods 8, $871-878$ (2011).

[4] M. S. Emara et al., IEEE Transactions on Biomedical Engineering 66, 1996-2009 (2019).

[5] S. Libbrecht et al., J. Neurophysiol. 120, 149-161 (2018).

[6] W. D. Sacher et al., bioRxiv 2020.2009.2030.317214 (2020).
[7] W. D. Sacher et al., Conference on Lasers and Electro-Optics, p. ATh4I.4 (2019).

[8] K. Van Acoleyen et al., IEEE Photonics Technol. Lett. 23,

1270-1272 (2011).

[9] J. Sun et al., Nature 493, 195-199 (2013).

[10] L. C. Moreaux et al., Neuron 108, 66 - 92 (2020). 